\title{
B physics with CMS upgraded detector
}

\author{
Fabrizio Palla*† \\ INFN Sezione di Pisa \\ E-mail: Fabrizio.Palla@cern.ch
}

The CMS potential for B-Physics with the Upgraded Phase-I and Phase-II detectors will be discussed, with the $\mathrm{B}_{s}^{0} \rightarrow \mu^{+} \mu^{-}$and $\mathrm{B}^{0} \rightarrow \mu^{+} \mu^{-}$benchmark channels, for the runs of the LHC at $\sqrt{s}=14 \mathrm{TeV}$ up to an integrated luminosity of $3000 \mathrm{fb}^{-1}$. With the upgraded CMS detector it will be possible to efficiently trigger and reconstruct both processes, with reduced statistical and systematic uncertainties leading to high precision measurements of the branching fractions of the $\mathrm{B}^{0} \rightarrow \mu^{+} \mu^{-}$and $\mathrm{B}_{s}^{0} \rightarrow \mu^{+} \mu^{-}$decays. This will allow in turn stringent tests of the Standard Model.

16th International Conference on B-Physics at Frontier Machines

2-6 May 2016

Marseille, France

${ }^{*}$ Speaker.

${ }^{\dagger}$ On behalf of CMS. 


\section{Introduction}

In the next years, the Large Hadron Collider (HL-LHC) will undergo into a series of improvements which should eventually be able to deliver to each general purpose experiment (ATLAS and CMS), a total integrated luminosity up to $\sim 3000 \mathrm{fb}^{-1}$ at a proton-proton collisions energy of 14 $\mathrm{TeV}$. This will be obtained through a much higher instantaneous luminosity with respect to the current LHC machine. In a first phase (Phase-I) doubling the nominal LHC instantaneous luminosity to $2 \times 10^{34} \mathrm{~cm}^{-2} \mathrm{~s}^{-1}$. In a second phase (Phase-II) the instantaneous luminosity will reach $5 \times 10^{34} \mathrm{~cm}^{-2} \mathrm{~s}^{-1}$ at the expenses of an average of 140 proton-proton interactions happening at every $25 \mathrm{~ns}$ in each collision point, requiring improvements in the detectors to cope with the new conditions. The high collected luminosity will allow to study rare processes at a sensitivity level never attained before, and the high collision energy will allow the study of particle types not normally produced at $\mathrm{B}$ factories. Analyses that will benefit from these factors include the search and study of $\mathrm{B} \rightarrow \mu^{+} \mu^{-}$decays or the search for lepton-flavor violation in $\mathrm{B}$ or $\tau$ decays $\left(\mathrm{B}^{0} \rightarrow \mu \tau\right.$, $\tau \rightarrow \mu \mu \mu)$

\section{CMS detector changes}

In Phase-I the new tracker detector will feature a lighter pixel system [1], made of 4 pixel barrel layers and 3 disks on either endcaps, to be deployed in 2017. The first measurement layer will be placed at about $3 \mathrm{~cm}$ from the beam, instead of the $4.4 \mathrm{~cm}$ of the current detector.

In the upgraded detector, support and services are redesigned to be lighter than the present system, using an ultra-lightweight support with $\mathrm{CO}_{2}$ cooling, and by relocating much of the passive material, like the electronic boards and connections, out of the tracking volume. The expected improvement in impact parameter resolution is between a factor of 1.2 to 1.6 better in the transverse plane, and between 1.5 to 2 in the longitudinal plane.

In Phase-II the CMS experiment will be completely refurbished [2], with enhancements to the muon system and a new inner tracker, with improved granularity, to cope with average occupancy resulting from 140 interactions expected per bunch crossing, and a completely new Trigger system. In particular, the new inner tracker system is expected to have L1-trigger tracking capabilities, in order to reconstruct efficiently all tracks with $p_{\mathrm{T}}>2 \mathrm{GeV}$. The muon system will also have improved coverage in the forward direction and extended trigger capabilities. The L1-Trigger and the High-Level Trigger (HLT) will increase their bandwidths to $750 \mathrm{MHz}$ and $7.5 \mathrm{kHz}$, respectively, almost one order of magnitude larger with respect to the current one. Also the L1-Trigger latency will be increased from the current 4 to $12.5 \mu \mathrm{s}$, in order to be able to incorporate the new information coming from the Silicon Tracker and exploit more complex algorithms as of today, allowing to retain the same physics capability for the high-pT processes as of LHC Run-1 and Run-2.

The usage of the charged tracks information (albeit with $p_{\mathrm{T}}>2 \mathrm{GeV}$ ) turns out to have important implications for B-physics. The pixel system will be made of 4 pixel barrel layers and a forward pixel coverage, consisting of 10 disks extending the coverage up to $|\eta| \approx 4$.0. The outer tracker material budget will diminish by roughly a factor of 2 in the central region $(|\eta|<1)$ and about a factor of 3 in the intermediate region around $1.2<|\eta|<1.5$. This, combined with 
a smaller silicon sensors pitch, will improve the momentum resolution especially in the "barrel" region $(|\eta|<1.4)$.

The quality of the tracks reconstructed at L1-Trigger is comparable to those reconstructed offline, as far as the momentum and angular resolutions is concerned; however the impact parameter cannot use the pixel detector information, hence resulting in a degraded resolution compared to the offline. Despite of this, the longitudinal impact parameter can be determined with an accuracy of about $1 \mathrm{~mm}$, which is of the same order of magnitude of the separation between two vertices in the 140 pileup bunch crossings.

Finally, CMS is currently exploring the possibility of a "timing" layer, with few pico-seconds resolution, capable to provide a time stamp to each track reconstructed in the Tracker. Albeit still no proposal has been formally presented, this would enhance the performances of CMS in reducing the ambiguity to assign tracks to the correct primary vertex, and some $\pi-\mathrm{K}$ separation up to 2-3 $\mathrm{GeV}$ in the barrel.

\section{B-Physics Studies with upgraded CMS detector}

So far, the impact on B-Physics of an upgraded CMS detector has been studied in detail only for the $\mathrm{B}_{s}^{0} \rightarrow \mu^{+} \mu^{-}$and $\mathrm{B}^{0} \rightarrow \mu^{+} \mu^{-}$benchmark channels. Other decay modes are being studied, including fully hadronic decays, thanks to the capability to efficiently reconstruct tracks above 2 $\mathrm{GeV}$ at the L1 Trigger level in Phase-II.

The main focus of the study is to quantify the ability to improve the measurements of the Branching Fractions thanks to an increased luminosity, by making usage of a Level-1 Track Trigger and a better momentum resolution of the Phase-II scenario, as compared to a Phase-I scenario. In order to focus only on the impact of the improved detector, for this study we have not considered the effects of any possible improvements on the analysis strategy. The published Run-1 analysis [3] was focused on achieving the maximum sensitivity for $\mathrm{B}_{s}^{0} \rightarrow \mu^{+} \mu^{-}$decays, thus it is conceivable that an analysis optimized for $\mathrm{B}^{0} \rightarrow \mu^{+} \mu^{-}$could obtain better results for that channel. For instance, different selections and fits, as well as additional normalization channels, could be used.

The study is based on the full detector simulation as far as the performance of the L1 trigger and of the offline reconstruction is concerned. Monte-Carlo samples of the $\mathrm{B}_{s}^{0} \rightarrow \mu^{+} \mu^{-}$signal with the simulation of the CMS detector for the Phase-I and Phase-II upgrade scenarios, including the Level-1 trigger emulation, were used. In addition, for the calculation of trigger rates, a minimumbias sample corresponding to the Phase-II scenario was also used. We take into account that the future runs of the LHC will be at an increased centre-of-mass energy, $14 \mathrm{TeV}$, by approximating that the $\mathrm{b} \overline{\mathrm{b}}$ cross section increases by $75 \%$ as compared to the $8 \mathrm{TeV}$ centre-of-mass cross section.

\subsection{L1 Trigger with Tracks}

A Level 1 trigger for the $\mathrm{B}^{0}$ and $\mathrm{B}_{s}^{0}$ signal is built exploiting the triggering capability of the upgraded CMS tracker. Events are triggered by asking for two opposite-charge muons, reconstructed matching the track-trigger track in the inner silicon detector with an outer track in the muon chambers.

Each muon is required to have transverse momentum $p_{\mathrm{T}}^{\mu}>3 \mathrm{GeV}$ and pseudorapidity $\left|\eta^{\mu}\right|<2$. In addition, the two muons are required to originate from the same primary vertex within $1 \mathrm{~cm}$. 
These selections are comparable with the ones employed in the published Run-1measurement. The Level $1 \mathrm{~B}$ candidate is built from the two muons and is required to have $p_{\mathrm{T}}^{\mu \mu}>4 \mathrm{GeV},\left|\eta^{\mu \mu}\right|<2$, and $3.9<M^{\mu \mu}<6.9 \mathrm{GeV}$.

The invariant mass distribution of the Level $1 \mathrm{~B}$ candidates for $\mathrm{B}^{0} \rightarrow \mu^{+} \mu^{-}$and $\mathrm{B}_{s}^{0} \rightarrow \mu^{+} \mu^{-}$signal events passing the trigger selections defined above is shown in Fig. 1 for an integrated luminosity of $3000 \mathrm{fb}^{-1}$. For $\mathscr{L}_{\text {int }}=3000 \mathrm{fb}^{-1}$, the number of expected L1 triggered signal events is equal to about 1700 for $\mathrm{B}^{0} \rightarrow \mu^{+} \mu^{-}$and about 16000 for $\mathrm{B}_{s}^{0} \rightarrow \mu^{+} \mu^{-}$. The invariant mass resolution at L1 is measured to be about $70 \mathrm{MeV}$ for both resonances. Due to the very low statistics, only

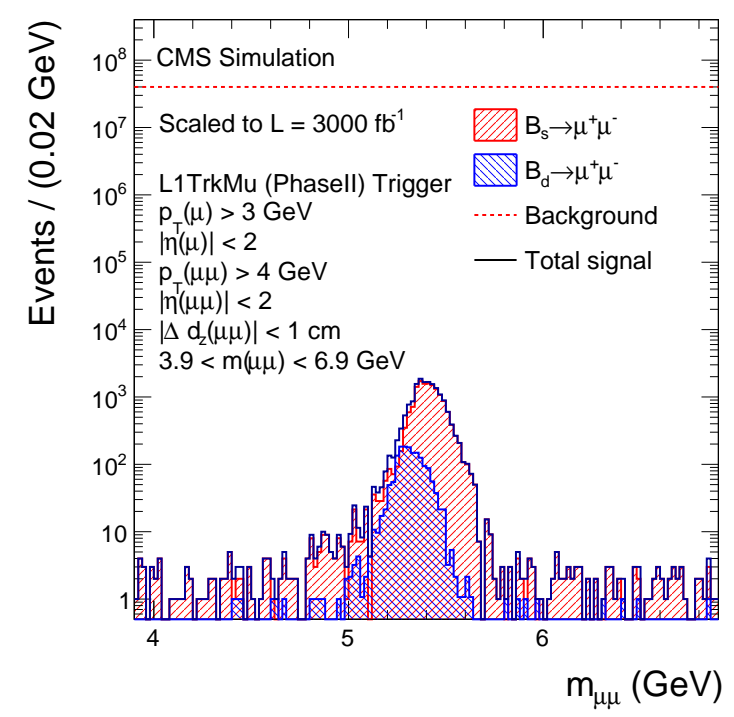

Figure 1: The di-muon invariant mass distributions at Level 1 trigger for $\mathrm{B}^{0} \rightarrow \mu^{+} \mu^{-}$(blue) and $\mathrm{B}_{s}^{0} \rightarrow$ $\mu^{+} \mu^{-}$(red) events for a total integrated luminosity of $3000 \mathrm{fb}^{-1}$. The red dashed line shows the expected background level.

an upper limit on the trigger rate can be assessed, equal to a few hundred $\mathrm{Hz}$. This corresponds to a small fraction of the total available $\mathrm{L} 1$ bandwidth (about $1 \mathrm{MHz}$ ), therefore it can be concluded that a low- $p$ T track-trigger-based algorithm for $\mathrm{B} \rightarrow \mu^{+} \mu^{-}$is expected to be entirely feasible for the Phase-II L1-trigger architecture.

\subsection{Effects of increased pileup}

With increasing instantaneous luminosity we anticipate increasing number of primary vertices per each bunch crossing. For Run-3 the better impact parameter resolution will help to handle the increased pile-up and we therefore do not expect significant impact on the analysis efficiency from this effect. For the Phase-II , the large number of primary vertices per bunch crossing (on average 140) will impact the variables used to discriminate the signal from the backgrounds, and in particular the isolation ones, due to a non negligible chance of a pile-up vertex being close to the B-meson production one, along the beam line. 
To evaluate the effects of the pile-up on offline reconstruction we study the distributions of the $\mathrm{B}_{s}^{0}$ candidate isolation discriminant in the Phase-II scenario, respectively with no simulated pile-up or with average pile-up of 140 events per bunch crossing. The definition of the B-candidate isolation variable is the same as in the Run-1analysis [3]

The normalized distributions of the isolation variable for the two pile-up scenarios are shown in Fig. 2. Adding 140 pile-up interactions lowers the peak at $I=1$ by about $16 \%$, therefore diminishing the discriminating power of this variable with respect to the combinatorial (not-isolated) background.

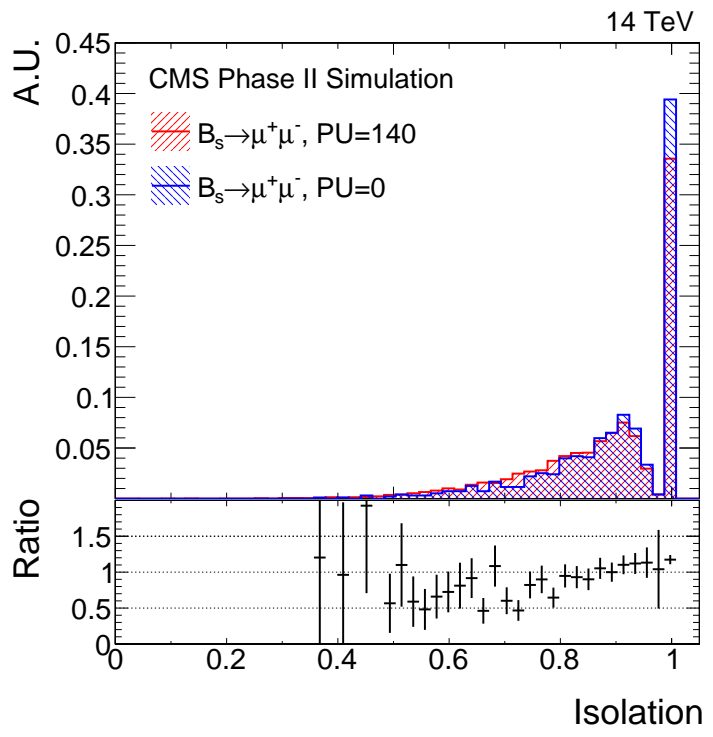

Figure 2: Normalized isolation variable distributions for the $\mathrm{B}_{s}^{0}$ signal for $\mathrm{PU}=0$ and $\mathrm{PU}=140$. The blue distribution represents the case with no pile-up while the red one is for average pile-up of 140 interactions per bunch crossing. In the bottom, the ratio between the $\mathrm{PU}=0$ and the $\mathrm{PU}=140$ distributions is also shown.

It should be stressed that the effect of the increased PU on the analysis efficiency is not limited to the isolation variable $I$, although the latter is arguably the most affected one. Indeed, with 140 pile-up events, the resulting larger number of tracks could potentially affect pattern recognition, requiring tightening of reconstruction criteria, to keep a level of fake tracks identical to what was estimated in the 2012 data (order of a percent). We have estimated a reduction of the single-muon tracking efficiency by $2.5 \%$. Based on the isolation study results shown above, and assuming a generous safety margin due to possible increase of the pileup (up to 200) or uncertainty in the longitudinal luminous profile, we have assumed in total a $35 \%$ loss of analysis efficiency for signal and a $30 \%$ less background rejection effectiveness.

Moreover, the typical resolution in the offline track longitudinal impact parameter in the endcaps $(0.5$ to $1 \mathrm{~mm}$ ) is expected to be comparable (if not larger) to the average pile-up vertex separation, which will tend to reduce the impact of the endcap region in the analysis. The $\mathrm{B}^{0} \rightarrow \mu^{+} \mu^{-}$sensitivity is not limited only by the signal yield, but also by the separation between $\mathrm{B}^{0}$ and $\mathrm{B}_{s}^{0}$ signal distributions. Thus, the mass resolution becomes the critical ingredient of the analysis. The barrel part of the detector provides a better mass measurement resolution than the 
endcap region: for this reason our Phase-II study focuses on the barrel region only. The systematics, considered in [3] is assumed to scale with luminosity. More details could be found in reference [4].

\section{Analysis performance}

The analysis performance is determined in the two scenarios corresponding to the Phase-I detector with an ageing corresponding to $1000 \mathrm{fb}^{-1}$ of integrated luminosity, and the Phase-II upgraded detector. Each of the two muons is required to have $p_{\mathrm{T}}^{\mu}>4 \mathrm{GeV}$, and $\left|\eta^{\mu}\right|<1.4$ for the Phase-II scenario $\left(\left|\eta^{\mu}\right|<2.4\right.$ for the Phase-I scenario). The B candidate is built from the two muons and is required to have $p_{\mathrm{T}}^{\mu \mu}>5 \mathrm{GeV}$ and $4.9<M^{\mu \mu}<5.9 \mathrm{GeV}$.

Results are found for several integrated luminosities, i.e. $20 \mathrm{fb}^{-1}, 100 \mathrm{fb}^{-1}$, and $300 \mathrm{fb}^{-1}$ for the Phase-I scenario, and $3000 \mathrm{fb}^{-1}$ for the Phase-II scenario. The result at $300 \mathrm{fb}^{-1}$ for the Phase-I scenario is given also for muons in the barrel only $\left(\left|\eta^{\mu}\right|<1.4\right)$ in order to allow for a better comparison with the Phase-II.

The estimation of the analysis performance is done using pseudo-experiments, generated with a Monte-Carlo technique starting from the baseline Run-1 PDFs for the signals and backgrounds, modified with the assumptions and simulation results summarized in Section 3. In all cases, the production cross sections and branching fractions predicted by the Standard Model are assumed for $\mathrm{B}^{0}$ and $\mathrm{B}_{s}^{0}$.

Figure 3 shows the Monte-Carlo projections of the $\mathrm{B}^{0}$ and $\mathrm{B}_{s}^{0}$ analysis results for the PhaseIãnd Phase-II scenarios. The left plot corresponds to barrel events and a total integrated luminosity of $300 \mathrm{fb}^{-1}$, while the right plot corresponds to barrel events and a total integrated luminosity of $3000 \mathrm{fb}^{-1}$. These figures show qualitatively that while in the Phase-I scenario the $\mathrm{B}^{0}$ peak is covered by the long resolution tail of the $\mathrm{B}_{s}^{0}$ resonance, in the Phase-II scenario the two peaks can be resolved due to the improved invariant mass resolution.

The quantitative estimate of the analysis performance, extracted from the pseudo-experiments, is shown in Table 1. The table shows, for all the values of the integrated luminosity considered, the number of reconstructed $\mathrm{B}_{s}^{0}$ and $\mathrm{B}^{0}$, the total uncertainties on the $\mathrm{B}_{s}^{0} \rightarrow \mu^{+} \mu^{-}$and $\mathrm{B}^{0} \rightarrow$ $\mu^{+} \mu^{-}$branching fractions, the sensitivity for detecting the $\mathrm{B}^{0}$ signal, and the uncertainty on the ratio of the two branching fractions.

\section{Conclusions}

We have shown that the CMS detector will be able to perform important contributions to the B-Physics at the high-luminosity upgrade of LHC, focusing on $\mathrm{B}^{0}\left[\mathrm{~B}_{s}^{0}\right] \rightarrow \mu^{+} \mu^{-}$decays.

The Phase-II upgrade of CMS will allow to design a trigger for the $\mathrm{B} \rightarrow \mu^{+} \mu^{-}$signal having an acceptance comparable to the one employed in LHC Run-1, with a manageable rate despite the huge increase in instantaneous luminosity of HL-LHC.

In the Phase-II scenario, also the overall performance of the analysis is expected to improve enough to allow for an observation of $\mathrm{B}^{0} \rightarrow \mu^{+} \mu^{-}$in excess of $5 \sigma$ within the expected lifespan of HL-LHC. Moreover, the better Phase-II detector performance will allow to measure the branching fraction $\mathscr{B}\left(\mathrm{B}^{0} \rightarrow \mu^{+} \mu^{-}\right)$and the ratio $\frac{\mathscr{B}\left(\mathrm{B}^{0} \rightarrow \mu^{+} \mu^{-}\right)}{\mathscr{B}\left(\mathrm{B}_{s}^{0} \rightarrow \mu^{+} \mu^{-}\right)}$with precisions of $18 \%$ and $21 \%$ respectively. 

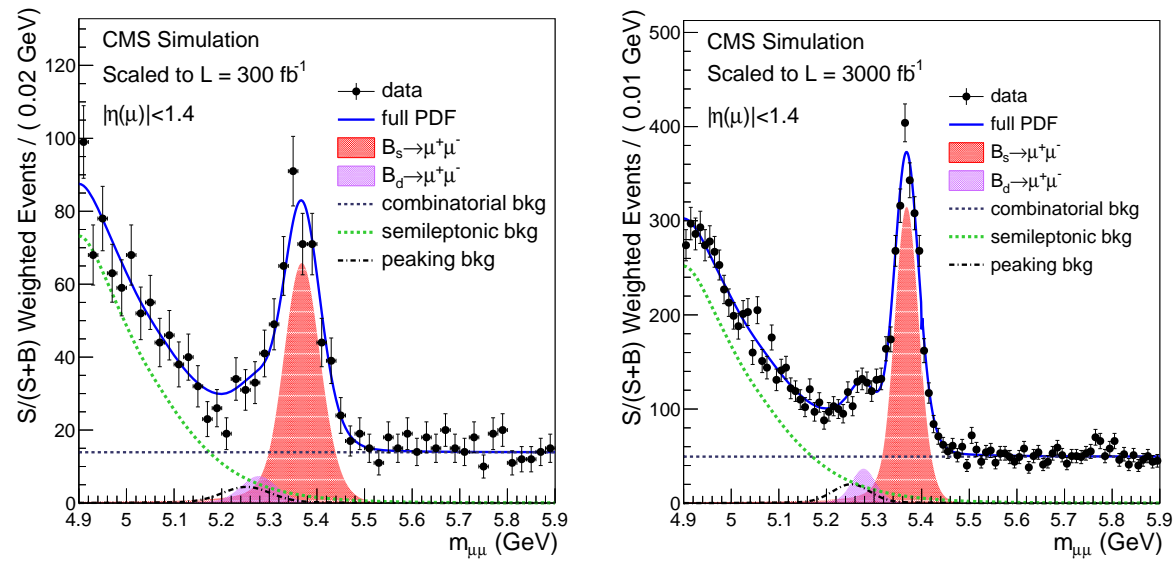

Figure 3: Projections of the mass fits to $300 \mathrm{fb}^{-1}$ (left) and $3000 \mathrm{fb}^{-1}$ (right) of integrated luminosity (L), respectively assuming the expected performances of Phase-I and Phase-II CMS detectors.

Table 1: The estimated analysis sensitivity from pseudo-experiments for different integrated luminosities. Columns in the table are, from left to right: the total integrated luminosity, the number of reconstructed $\mathrm{B}_{s}^{0}$ and $\mathrm{B}^{0}$, the total uncertainties on the $\mathrm{B}_{s}^{0} \rightarrow \mu^{+} \mu^{-}$and $\mathrm{B}^{0} \rightarrow \mu^{+} \mu^{-}$branching fractions, the $\mathrm{B}^{0}$ statistical significance, and uncertainty on the ratio between the branching fractions. Results up to $300 \mathrm{fb}^{-1}$ are for the Phase-I scenario, whereas the result for $3000 \mathrm{fb}^{-1}$ is for the Phase-II.

Estimate of analysis sensitivity

\begin{tabular}{l|l|l|l|l|l|l}
$\mathscr{L}\left(\mathrm{fb}^{-1}\right)$ & $N\left(\mathrm{~B}_{s}^{0}\right)$ & $N\left(\mathrm{~B}^{0}\right)$ & $\delta \mathscr{B}\left(\mathrm{B}_{s}^{0} \rightarrow \mu^{+} \mu^{-}\right)$ & $\delta \mathscr{B}\left(\mathrm{B}^{0} \rightarrow \mu^{+} \mu^{-}\right)$ & $\mathrm{B}^{0}$ sign. & $\delta \frac{\mathscr{B}\left(\mathrm{B}^{0} \rightarrow \mu^{+} \mu-\right.}{\mathscr{B}\left(\mathrm{B}_{s}^{0} \rightarrow \mu^{+} \mu_{\top}^{-}\right)}$ \\
\hline 20 & 18.2 & 2.2 & $35 \%$ & $>100 \%$ & $0.0-1.5 \sigma$ & $>100 \%$ \\
100 & 159 & 19 & $14 \%$ & $63 \%$ & $0.6-2.5 \sigma$ & $66 \%$ \\
300 & 478 & 57 & $12 \%$ & $41 \%$ & $1.5-3.5 \sigma$ & $43 \%$ \\
300 (barrel) & 346 & 42 & $13 \%$ & $48 \%$ & $1.2-3.3 \sigma$ & $50 \%$ \\
3000 (barrel) & 2250 & 271 & $11 \%$ & $18 \%$ & $5.6-8.0 \sigma$ & $21 \%$
\end{tabular}

This will constitute a much more stringent test of Standard Model predictions than what will be possible with the Phase-ICMS detector.

\section{References}

[1] CMS Collaboration, Technical proposal for the upgrade of the CMS detector through 2020, CERN-LHCC-2011-006. LHCC-P-004, Jun. 2011.

[2] CMS Collaboration, Technical Proposal for the Phase-II Upgrade of the CMS Detector, LHCC-P-008, Jun. 2015".

[3] S. Chatrchyan et al. (CMS Collaboration), "Measurement of the $\mathrm{B}_{s}^{0} \rightarrow \mu^{+} \mu^{-}$branching fraction and search for $\mathrm{B}^{0} \rightarrow \mu^{+} \mu^{-}$with the CMS experiment", Phys. Rev. Lett., 111, 101804, (2013). 
[4] CMS Collaboration, "B Physics analyses for the Phase-II Upgrade Technical Proposal", CMS-PAS-FTR-14-015, 2015. 